\title{
A nonlinear nonautonomous delay differential inequality for dissipativity of Lotka-Volterra functional differential equations
}

\author{
Liguang $\mathrm{Xu}^{a, *}$, Danhua $\mathrm{He}^{b}$ \\ ${ }^{a}$ Department of Applied Mathematics, Zhejiang University of Technology, Hangzhou, 310023, PR China \\ ${ }^{b}$ Department of Mathematics, Zhejiang International Studies University, Hangzhou, 310012, PR China \\ * Corresponding Author \\ E-mail: xlg132@126.com
}

\begin{abstract}
In this paper, a Lotka-Volterra functional differential equation is considered. By establishing a nonlinear nonautonomous delay differential inequality and using a generalized Barbălat's lemma, we obtain some new sufficient conditions ensuring the dissipativity of the Lotka-Volterra functional differential equation.
\end{abstract}

2000 Mathematics Subject Classification. 34K38. 34K25.

Keywords. Lotka-Volterra functional differential equations, Nonlinear delay differential inequality, Dissipativity, Generalized Barbălat's lemma.

\section{Introduction}

Let $H$ be a real or complex Hilbert space with the inner product $\langle\cdot, \cdot\rangle$ and the corresponding norm $\|\cdot\|, X$ be a dense continuously imbedded subspace of $\mathrm{H}$. For any given closed interval $I \subset R$, let the symbol $C_{X}(I)$ denote a Banach space consisting of all continuous mappings $x: I \rightarrow X$, on which the norm is defined by $\|x\|_{\infty}=\max _{t \in I}\|x(t)\|$. Consider the initial value problem in Lotka-Volterra functional differential equations

$$
\left\{\begin{array}{l}
y^{\prime}(t)=g(t, y(t), y(\cdot))=\eta(t, y(t)) f(t, y(t), y(\cdot)), t \geq 0 \\
y(t)=\varphi(t),-\tau \leq t \leq 0
\end{array}\right.
$$

where $\tau$ is a positive constant, $\varphi \in C_{X}[-\tau, 0]$ is a given intial function, $\eta:[0,+\infty) \times X \rightarrow H$ is a nonnegative continuous function, $f:[0,+\infty) \times X \times C_{X}[-\tau,+\infty) \rightarrow H$, and $g:[0,+\infty) \times X \times$ $C_{X}[-\tau,+\infty) \rightarrow H$ is a given locally Lipschitz continuous mapping satisfying

$$
\begin{aligned}
& 2 \mathfrak{R}<u, g(t, u, \psi(\cdot))>\leq \eta(t, u)\left[\gamma(t)+\alpha(t)\|u\|^{2}+\beta(t) \max _{t-\mu_{2}(t) \leq \theta \leq t-\mu_{1}(t)}\|\psi(\theta)\|^{2}\right], \\
& u \in X, \psi \in C_{X}[-\tau,+\infty), t \in[0,+\infty)
\end{aligned}
$$

where the functions $\mu_{1}(t)$ and $\mu_{2}(t)$ are assumed to satisfy

$$
0 \leq \mu_{1}(t) \leq \mu_{2}(t) \leq t+\tau, \forall t \in[0,+\infty)
$$

$\alpha(t)$ and $\beta(t)$ are continuous functions and $\gamma(t)$ is a bounded continuous functions on the interval $[0,+\infty)$. 
Recently, Wen, Yu and Wang [1] discussed the dissipativity of (1) with $\eta(t, y(t)) \equiv 1$. They established the generalized Halanay inequality and obtained the dissipativity results of (1) with $\eta(t, y(t)) \equiv 1$. In this paper, we will improve the inequality in [1] such that it is effective for $(1)$. By establishing a nonlinear nonautonomous delay differential inequality and using a generalized Barbălat's lemma, we obtain some new sufficient conditions ensuring the dissipativity of (1).

\section{Nonlinear delay differential inequality}

Theorem 2.1. If $y(t) \geq 0$ is a differentiable function defined on $(-\infty,+\infty)$, and

$$
\left\{\begin{array}{l}
u^{\prime}(t) \leq \eta(t, y(t))\left[\gamma(t)+\alpha(t) u(t)+\beta(t) \sup _{t-\tau(t) \leq \theta \leq t} u(\theta)\right], t \geq t_{0} \\
y(t)=\psi(t), t \leq t_{0}
\end{array}\right.
$$

where $u(t)=\|y(t)\|^{2}$, and $\psi(t)$ is bounded and continuous for $t \leq t_{0}$, continuous functions $\gamma(t) \geq 0$, $\beta(t) \geq 0$ and $\alpha(t) \leq 0$ for $t \in\left[t_{0},+\infty\right), \tau(t) \geq 0, \eta:[0,+\infty) \times X \rightarrow H$ is a nonnegative continuous function, and if there exists $\sigma>0$ such that

$$
\alpha(t)+\beta(t) \leq-\sigma<0 \text { for } t \geq t_{0},
$$

then we have

(i)

$$
u(t) \leq \frac{\gamma^{*}}{\sigma}+G, t \geq t_{0}
$$

(ii)

$$
u(t) \leq \frac{\gamma^{*}}{\sigma}+G e^{-\mu^{*} \int_{t_{0}}^{t} \eta(s, y(s)) d s}, t \geq t_{0},
$$

where $G=\sup _{-\infty<\theta \leq t_{0}}\|\psi(\theta)\|^{2}, \gamma^{*}=\sup _{t_{0} \leq t<+\infty} \gamma(t)$, and $\mu^{*} \geq 0$ is defined as

$$
\mu^{*}=\inf _{t \geq t_{0}}\left\{\mu(t): \mu(t)+\alpha(t)+\beta(t) e^{h \mu(t) \tau(t)}=0\right\}
$$

where

$$
h=\sup _{t \geq t_{0}} \max _{(s, u) \in[t-\tau, t] \times\left[0, \frac{\gamma^{*}}{\sigma}+G\right]} \eta(t, y)<\infty .
$$

Proof. (i): We at first shall prove that for any positive constant $\varepsilon$,

$$
u(t) \leq \frac{\gamma^{*}+\varepsilon}{\sigma}+G, t \geq t_{0}
$$

If (10) does not hold, then there exists $t_{1}>t_{0}$ such that

$$
u\left(t_{1}\right)=\frac{\gamma^{*}+\varepsilon}{\sigma}+G, u^{\prime}\left(t_{1}\right)>0, u(t) \leq \frac{\gamma^{*}+\varepsilon}{\sigma}+G, t \in\left(-\infty, t_{1}\right] .
$$


Using (4), (5) and (11), we obtain that

$$
\begin{aligned}
u^{\prime}\left(t_{1}\right) & \leq \eta\left(t_{1}, y\left(t_{1}\right)\right)\left[\gamma\left(t_{1}\right)+\alpha\left(t_{1}\right) u\left(t_{1}\right)+\beta\left(t_{1}\right) \sup _{t_{1}-\tau\left(t_{1}\right) \leq \theta \leq t_{1}} u(\theta)\right] \\
& \leq \eta\left(t_{1}, y\left(t_{1}\right)\right)\left[\gamma^{*}+\alpha\left(t_{1}\right)\left(\frac{\gamma^{*}+\varepsilon}{\sigma}+G\right)+\beta\left(t_{1}\right)\left(\frac{\gamma^{*}+\varepsilon}{\sigma}+G\right)\right] \\
& \leq \eta\left(t_{1}, y\left(t_{1}\right)\right)\left[\frac{\gamma^{*}+\varepsilon}{\sigma}\left(\sigma+\alpha\left(t_{1}\right)+\beta\left(t_{1}\right)\right)+G\left(\alpha\left(t_{1}\right)+\beta\left(t_{1}\right)\right]\right. \\
& \leq-\eta\left(t_{1}, y\left(t_{1}\right)\right) \sigma G \leq 0 .
\end{aligned}
$$

This contradicts the inequality in (11), and so (10) holds. Since $\varepsilon>0$ is arbitrary, we let $\varepsilon \rightarrow 0$ and obtain (6).

(ii): By (6), one can know that the definition of $h$ for (9) is reasonable. Denote

$$
H(\mu)=\mu+\alpha(t)+\beta(t) e^{h \mu \tau(t)} .
$$

For any fixed $t \geq t_{0}$, we see that

$$
H(0)=\alpha(t)+\beta(t) \leq-\sigma<0, \lim _{\mu \rightarrow+\infty} H(\mu)=+\infty
$$

and

$$
H^{\prime}(\mu)=1+\tau(t) \beta(t) h e^{\mu h \tau(t)}>0
$$

Therefore for any given $t \geq t_{0}$ there is a unique positive $\mu$ such that

$$
\mu+\alpha(t)+\beta(t) e^{h \mu \tau(t)}=0
$$

that means the (16) define an implicit function $\mu(t)$ for $t \geq t_{0}$. From that definition, one has $\mu^{*} \geq 0$. Next, we at first shall prove that for any positive constant $\varepsilon$,

$$
u(t) \leq \frac{\gamma^{*}+\varepsilon}{\sigma}+G e^{-\mu^{*} \int_{t_{0}}^{t} \eta(s, y(s)) d s} \triangleq v(t), t \geq t_{0} .
$$

If (17) is not true, then there exists a constant $\xi>t_{0}$ such that

$$
u(\xi)=v(\xi), u^{\prime}(\xi)>v^{\prime}(\xi), u(t) \leq v(t), t \in\left[t_{0}, \xi\right) .
$$

Let $w(t)=v(t)-u(t)$, then we have

$$
\begin{aligned}
w^{\prime}(\xi) & =v^{\prime}(\xi)-u^{\prime}(\xi) \\
& \geq-G \mu^{*} \eta(\xi, y(\xi)) e^{-\mu^{*} \int_{t_{0}}^{\xi} \eta(s, y(s)) d s}-\eta(\xi, y(\xi))\left[\gamma(\xi)+\alpha(\xi) u(\xi)+\beta(\xi) \sup _{\xi-\tau(\xi) \leq \theta \leq \xi} u(\theta)\right] \\
& >-G \mu^{*} \eta(\xi, y(\xi)) e^{-\mu^{*} \int_{t_{0}}^{\xi} \eta(s, y(s)) d s}-\eta(\xi, y(\xi))\left[\gamma^{*}+\varepsilon+\alpha(\xi) u(\xi)+\beta(\xi) \sup _{\xi-\tau(\xi) \leq \theta \leq \xi} u(\theta)\right] .
\end{aligned}
$$


If $\xi-\tau(\xi) \geq t_{0}$, it follows from (19) that

$$
\begin{aligned}
w^{\prime}(\xi) \geq & -G \mu^{*} \eta(\xi, y(\xi)) e^{-\mu^{*} \int_{t_{0}}^{\xi} \eta(s, y(s)) d s}-\eta(\xi, y(\xi))\left[\gamma^{*}+\varepsilon+\alpha(\xi)\left(\frac{\gamma^{*}+\varepsilon}{\sigma}+G e^{-\mu^{*} \int_{t_{0}}^{\xi} \eta(s, y(s)) d s}\right)\right. \\
& \left.+\beta(\xi)\left(\frac{\gamma^{*}+\varepsilon}{\sigma}+G e^{-\mu^{*} \int_{t_{0}}^{\xi-\tau(\xi)} \eta(s, y(s)) d s}\right)\right] \\
= & \eta(\xi, y(\xi))\left[-\frac{\gamma *+\varepsilon}{\sigma}(\sigma+\alpha(\xi)+\beta(\xi))-G e^{-\mu^{*} \int_{t_{0}}^{\xi} \eta(s, y(s)) d s}\left(\mu^{*}+\alpha(\xi)+\beta(\xi) e^{\mu^{*} \int_{\xi-\tau(\xi)}^{\xi} \eta(s, y(s)) d s}\right) .\right.
\end{aligned}
$$

From the define of function $\mu(t)$, we have

$$
\begin{aligned}
\mu^{*}+\alpha(\xi) & +\beta(\xi) e^{\mu^{*} \int_{\xi-\tau(\xi)}^{\xi} \eta(s, y(s)) d s} \\
& =\mu^{*}+\alpha(\xi)+\beta(\xi) e^{\mu^{*} \int_{\xi-\tau(\xi)}^{\xi} \eta(s, y(s)) d s}-\mu(\xi)-\alpha(\xi)-\beta(\xi) e^{h \mu(\xi) \tau(\xi)} \\
& =\left(\mu^{*}-\mu(\xi)\right)+\beta(\xi)\left(e^{\mu^{*} \int_{\xi-\tau(\xi)}^{\xi} \eta(s, y(s)) d s}-e^{h \mu(\xi) \tau(\xi)}\right) \leq 0
\end{aligned}
$$

Noting (5), therefore (20) yields

$$
w^{\prime}(\xi)=v^{\prime}(\xi)-u^{\prime}(\xi) \geq 0,
$$

which contradicts the first inequality in (18).

If $\xi-\tau(\xi)<t_{0}$, it follows from (19) that

$$
\begin{aligned}
w^{\prime}(\xi) \geq & -G \mu^{*} \eta(\xi, y(\xi)) e^{-\mu^{*} \int_{t_{0}}^{\xi} \eta(s, y(s)) d s}-\eta(\xi, y(\xi))\left[\gamma^{*}+\varepsilon+\alpha(\xi)\left(\frac{\gamma^{*}+\varepsilon}{\sigma}+G e^{-\mu^{*} \int_{t_{0}}^{\xi} \eta(s, y(s)) d s}\right)\right. \\
& \left.+\beta(\xi) \max \left\{\sup _{\theta \leq t_{0}} u(\theta), \sup _{t_{0} \leq \theta \leq \xi} u(\theta)\right\}\right] \\
\geq & -G \mu^{*} \eta(\xi, y(\xi)) e^{-\mu^{*} \int_{t_{0}}^{\xi} \eta(s, y(s)) d s}-\eta(\xi, y(\xi))\left[\gamma^{*}+\varepsilon+\alpha(\xi)\left(\frac{\gamma^{*}+\varepsilon}{\sigma}+G e^{-\mu^{*} \int_{t_{0}}^{\xi} \eta(s, y(s)) d s}\right)\right. \\
& \left.+\beta(\xi)\left(G+\frac{\gamma^{*}+\varepsilon}{\sigma}\right)\right] \\
= & \eta(\xi, y(\xi))\left[-\frac{\gamma^{*}+\varepsilon}{\sigma}(\sigma+\alpha(\xi)+\beta(\xi))-G e^{-\mu^{*} \int_{t_{0}}^{\xi} \eta(s, y(s)) d s}\left(\mu^{*}+\alpha(\xi)+\beta(\xi) e^{\mu^{*} \int_{t_{0}}^{\xi} \eta(s, y(s)) d s}\right)\right. \\
\geq & \eta(\xi, y(\xi))\left[-\frac{\gamma^{*}+\varepsilon}{\sigma}(\sigma+\alpha(\xi)+\beta(\xi))-G e^{-\mu^{*} \int_{t_{0}}^{\xi} \eta(s, y(s)) d s}\left(\mu^{*}+\alpha(\xi)+\beta(\xi) e^{\mu^{*} \int_{\xi-\tau(\xi)}^{\xi} \eta(s, y(s)) d s}\right) .\right.
\end{aligned}
$$

Here we also obtain that (22) holds, which contradicts the first inequality in (18). Hence the inequality (17) must hold. Since $\varepsilon>0$ is arbitrary, we let $\varepsilon \rightarrow 0$ and obtain (7). The proof of Theorem 2.1 is completed.

Remark 2.2. Suppose that $\eta(t, y(t)) \equiv 1$ in Theorem 2.1, then we get Theorem 2.4 in [1].

\section{Dissipativity of Lotka-Volterra functional differential equations}

Definition 3.1. (See [1]) System (1) is said to be dissipative in $H$ if there exists a bounded set $B \subset H$, such that for any given bounded set $\Phi \subset H$, there is a time $t^{*}=t^{*}(\Phi)$, such that for 
any given initial function $\varphi \in C_{X}[-\tau, 0]$ with $\varphi$ contained in $\Phi$ for all $t \in[-\tau, 0]$, the values of the corresponding solution $y(t)$ of the problem are contained in $B$ for all $t \geq t^{*}$. Here $B$ is called an absorbing set of the problem.

Lemma 3.2. (Generalized Barbălat's lemma [2]) If

$\left(H_{1}\right) u: \mathbb{R}_{+} \rightarrow \mathbb{R}^{n}$ is uniformly continuous;

$\left(H_{2}\right) g: \mathbb{R}^{n} \rightarrow \mathbb{R}$ is continuous and $g(x)=0$ iff $x=0$;

$\left(H_{3}\right) h: \mathbb{R}_{+} \rightarrow \mathbb{R}_{+}$satisfies $\mathcal{K}(\delta) \triangleq \inf _{t \geq 0} \int_{t}^{t+\delta} h(s) d s>0$, for any $\delta>0 ;$

$\left(H_{4}\right) \lim _{t \rightarrow \infty} \int_{0}^{t} h(s) g(u(s)) d s$ exists and is finite;

then $\lim _{t \rightarrow \infty} u(t)=0$.

Theorem 3.3. Suppose that $y(t)$ is a solution of the problem (1) satisfying the condition (2), and there exists a constant $\sigma>0$ such that

$$
\alpha(t)+\beta(t) \leq-\sigma<0 \text { for } t \geq 0
$$

Then

(i)

$$
\|y(t)\|^{2} \leq \frac{\bar{\gamma}^{*}}{\sigma}+\bar{G}, t \geq 0
$$

(ii)

$$
\|y(t)\|^{2} \leq \frac{\bar{\gamma}^{*}}{\sigma}+\bar{G} e^{-\mu^{*} \int_{t_{0}}^{t} \eta(s, y(s)) d s}, t \geq 0
$$

where $\bar{G}=\sup _{-\infty<\theta \leq 0}\|\varphi(\theta)\|^{2}, \bar{\gamma}^{*}=\sup _{0 \leq t<+\infty} \gamma(t)$, and $\bar{\mu}^{*} \geq 0$ is defined as

$$
\bar{\mu}^{*}=\inf _{t \geq 0}\left\{\mu(t): \mu(t)+\alpha(t)+\beta(t) e^{h \mu(t) \tau(t)}=0\right\},
$$

where

$$
\bar{h}=\sup _{t \geq 0} \max _{\left(s,\|y\|^{2}\right) \in[t-\tau, t] \times\left[0, \frac{\bar{\gamma}^{*}}{\sigma}+\bar{G}\right]} \eta(t, y)<\infty .
$$

Proof. To apply the result of Theorem 2.1, we have to extend the define of initial function in (1) as $y(t)=\varphi(-\tau)$ for $-\infty<t \leq \tau$.

Let

$$
u(t)=\|y(t)\|^{2}=<y(t), y(t)>.
$$

From (2), we have

$$
\begin{aligned}
u^{\prime}(t) & =\frac{d}{d t}<y(t), y(t)>=2 \Re<y(t), g(t, y(t), y(\cdot))> \\
& \leq \eta(t, y(t))\left[\gamma(t)+\alpha(t) u(t)+\beta(t) \max _{t-\mu_{2}(t) \leq \theta \leq t-\mu_{1}(t)} u(\theta)\right] \\
& \leq \eta(t, y(t))\left[\gamma(t)+\alpha(t) u(t)+\beta(t) \max _{t-\mu_{2}(t) \leq \theta \leq t} u(\theta)\right] .
\end{aligned}
$$


Application of Theorem 2.1 to the above inequality yields (25) and (26). The proof is completed.

Corollary 3.4. In addition to the conditions of Theorem 3.3 hold, further assume that $\eta(s, y(s)) \geq$ $\delta>0$. Then,

(i) for any given $\varepsilon>0$, there exists a positive number $t^{*}\left(\|\varphi\|_{\infty}, \varepsilon\right)$, such that

$$
\|y(t)\|^{2} \leq \frac{\bar{\gamma}^{*}}{\sigma}+\varepsilon, \forall t>t^{*} .
$$

(ii) For any given $\varepsilon>0$, the problem (1) is dissipative with an absorbing set $B=B\left(0, \sqrt{\frac{\bar{\gamma}^{*}}{\sigma}+\varepsilon}\right)$.

Theorem 3.5: In addition to the conditions of Theorem 3.3 hold, further assume that $\eta(s, y(s))=$ $h(s) g(y(s))$, where $g$ and $h$ satisfy $\left(H_{2}\right)$ and $\left(H_{3}\right)$ of Lemma 3.2, respectively. Then, for any given $\varepsilon>0$, the problem (1) is dissipative with an absorbing set $B=B\left(0, \sqrt{\left.\frac{\bar{\gamma}^{*}}{\sigma}+\varepsilon\right)}\right.$.

Proof: We only need to consider the following two possible cases:

(i) If $\int_{0}^{\infty} \eta(s, y(s)) \mathrm{d} s=\infty$, then from (26) we have $\lim _{t \rightarrow \infty}\|y(t)\| \leq \sqrt{\frac{\bar{\gamma}^{*}}{\sigma}}$.

(ii) If $\int_{0}^{\infty} \eta(s, y(s)) \mathrm{d} s<\infty$, then $h(s) g(y(s)) \in L[0, \infty)$. From (25) and (30), we know that $\dot{y}(t)$ is bounded. So $y(t)$ is a uniformly continuous function. By Lemma 3.2, we have $\lim _{t \rightarrow \infty} y(t)=0 \leq$ $\sqrt{\frac{\bar{\gamma}^{*}}{\sigma}}$.

From above (i) and (ii), we know the problem (1) is dissipative with an absorbing set $B=$ $B\left(0, \sqrt{\frac{\bar{\gamma}^{*}}{\sigma}+\varepsilon}\right)$. The proof is completed.

Corollary 3.6. In addition to the conditions of Theorem 3.3 hold. If $\eta(s, y(s))=g(y(s))$, where $g(\cdot)$ is a continuous, positive definite function, then for any given $\varepsilon>0$, system (1) is dissipative with an absorbing set $B=B\left(0, \sqrt{\frac{\bar{\gamma}^{*}}{\sigma}+\varepsilon}\right)$.

Remark 3.7. In the recent years, various generalized Halanay inequalities have been established and successfully applied to the problem of investigating the dissipativity of differential systems, [1,3-6]. However, the generalized Halanay inequalities in [1,3-6] are ineffective for studying the dissipativity of (1) due to the existence of the term " $\eta(t, y(t))$ " of $(1)$, unless one resorts to the rather restrictive condition that $\eta(t, y(t)) \geq \delta>0$ ( $\delta$ is a constant).

\section{Acknowledgement}

The work is supported by National Natural Science Foundation of China (Grant No. 11101367) and the China Scholarship Council (Grant No. 201208330001).

\section{References}

[1] L.P. Wen, Y.X. Yu and W.S. Wang, Generalized Halanay inequalities for dissipativity of Volterra functional differential equations, J. Math. Anal. Appl. 347 (2008), 169-178. 
[2] D.Y. Xu and L.G. Xu, New results for studying a certain class of nonlinear delay differential systems, IEEE Trans. Autom. Control 55 (2010), 1641-1645.

[3] L.P. Wen, W.S. Wang and Y.X. Yu, Dissipativity and asymptotic stability of nonlinear neutral delay integro-differential equations, Nonlinear Anal. 72 (2010), 1746-1754.

[4] H.J. Tian, Numerical and analytic dissipativity of the h-method for delay differential equations with a bounded variable lag, Int. J. Bifur. Chaos 14 (2004), 1839-1845.

[5] S.Q. Gan, Dissipativity of linear $\theta$-methods for integro-differential equations, Comput. Math. Appl. 52 (2006), 449-458.

[6] S.Q. Gan, Dissipativity of $\theta$-methods for nonlinear Volterra delay-integro-differential equations, J. Comput. Appl. Math. 206 (2007), 898-907. 